\title{
Interaction Techniques and Vessel Analysis for Preoperative Planning in Liver Surgery
}

\author{
Bernhard Preim $^{1}$, Dirk Selle ${ }^{1}$, Wolf Spindler ${ }^{1}$, Karl J. Oldhafer $^{2}$, \\ Heinz-Otto Peitgen ${ }^{1}$ \\ ${ }^{1} \mathrm{MeVis}-$ Center for Medical Diagnostic Systems and Visualization, Universitätsallee 29, \\ 28359 Bremen, Germany, \\ \{preim, selle, spindler, peitgen\}@mevis.de \\ ${ }^{2}$ Universitätsklinikum Essen, Klinik für Allgemein- und Transplantationschirurgie, \\ Hufelandstr. 55, 45122 Essen, Germany, \\ karl.oldhafer@uni-essen.de
}

\begin{abstract}
We present visualization and interaction techniques for preoperative planning in oncologic liver surgery. After several image processing steps a $3 \mathrm{~d}$ visualization of all relevant anatomic and pathologic structures is created. In this $3 \mathrm{~d}$ visualization a surgeon can flexibly specify resection regions with resection tools which can be applied selectively to different structures. The combination of several views which can be synchronized makes it easy to compare different views on the resection plan.

In addition, we present the application of vessel analysis techniques in order to make suggestions for optimal resections according to guidelines for liver surgery. The basic idea for these suggestions is to define the region which has to be removed in order to resect a lesion with a given tumor free margin. For this purpose, the vessels involved and the region supplied by them is estimated. It turned out that the resections suggested provide a reasonable and useful basis for preoperative planning. This contribution presents novel methods which have not been evaluated thoroughly yet.
\end{abstract}

Keywords: computer-assisted surgery, vessel analysis, visualization

\section{Introduction}

Liver carcinoma belong to the most wide-spread malignant diseases world-wide. Among the well-established therapies surgical intervention is the only one which can have a curative effect ( 5 -year survival rate $\approx 30 \%$ ). Long-term survival depends on whether all lesions have been removed entirely with a sufficient tumor free margin. Therefore it is essential to decide on a reliable base whether a tumor can be treated surgically and which region should be resected. Currently, this decision is made on the base of planar slices of CT- and MR-images. This raises several problems: the spatial relationships between vessels and lesions are difficult to judge. The volume of lesions, the vessels involved in the resection of a lesion and the region which is supplied by the involved vessels can only roughly be estimated. Furthermore, it is often difficult to decide whether multiple lesions should be resected separately.

In this paper, we present the SURGERYPLANNER which is dedicated to liver surgery and provides decision support for the above-mentioned questions. The SURGERYPLANNER is based on our long-term project on image processing and analysis of CT liver images [4], [9], [10]. The SURGERYPLANNER contains three main components: 
- a flexible $3 \mathrm{~d}$ visualization for the exploration of the previously identified structures,

- a resection planning module which provides resection tools in order to try a resection strategy (e.g. to simulate the removal of parts of the liver) and

- a module which suggests resection regions on the background of guidelines for tumor-free margins and an analysis of the involved intrahepatic vessels.

Besides supporting the surgeon to preoperatively decide on the optimal resection strategy the SURGERYPLANNER is useful to discuss the intervention and to explain it to a patient.

\section{Medical Background}

The main issue of liver surgery is to take into account the individual intrahepatic vessel anatomy. The anatomy of the liver is characterized by four hierarchical vessel systems: the portal venous system, the liver veins, the arteries and the bile ducts. For preoperative planning the portal venous system plays a key role as it defines the functional units of the liver. Hepatic veins are also essential as they drain the liver. Bile ducts and arteries are very close to the portal veins and are of minor importance for surgery planning.

Following the Couinaud model [2] which is wide-spread in Europe the liver is divided into segments which are defined according to the branching structure of the portal vein. A segment is supplied by a third-order portal vein branch. The hepatic veins proceed between the different segments. Liver segments are highly variable from patient to patient in shape and size [4]. Even the number of segments may be different. As the boundaries between the segments can not be localized by external landmarks it is impossible to identify segments exactly during an operation. In this paper we describe methods to support liver surgery by preoperatively identifying liver segments and subsegments as suitable resection regions. The prerequisites for this purpose are the segmentation of the liver and of all lesions as well as the segmentation of the portal venous system and of hepatic veins.

In order to get a better understanding of the requirements surgeons actually have in practice, we have polled liver surgeons by sending out some 30 questionaires from which 11 have been returned (all 11 are male surgeons, average age 40 years, experience in tumor surgery on the average 10 years). All surgeons indicated that the spatial relations between vessels and lesions are difficult to judge and that a $3 \mathrm{~d}$ visualization and quantitative analysis would be helpful for this purpose. Almost all, 10 of the 11 surgeons indicated they would appreciate trying out resection strategies preoperatively which reveals that it is often not obvious how to resect a lesion. Based on the survey we developed the following scenario which guides our development: a surgeon selects a lesion and specifies a safety margin (in liver surgery 10 or $15 \mathrm{~mm}$ are considered appropriate). The system visualizes the margin and highlights all vessels involved. Beside the directly involved vessels the peripheral part of this vessel system (starting from the directly involved vessels) is highlighted to indicate that these would be destroyed. Finally, it is estimated which region of the liver parenchyma is supplied by the involved vessels because this region should be 
removed. In addition, the volume of the resection region (percentage of the total liver volume) is displayed. If too much tissue would be removed the safety margin can be decreased to find out whether the resection is possible with a $5 \mathrm{~mm}$ margin (which reduces the long-term survival expectance but is still considerably better than no operation at all).

\section{Prior and Related Work}

Computer support for planning liver resections is challenging. The identification and delineation of the liver and of the lesions inside it, as well as the vessel segmentation requires excellent radiological data and a variety of dedicated and robust methods which still form an area of active research. Several complex image processing steps are needed to come up with a $3 \mathrm{~d}$ model for surgery planning. In [5] the problems are described and a particular problem - transfer function design to highlight the liver and the vessels in a volume visualization - has been dealt with. Another particular problem, the liver vessel analysis, has been tackled in [3] where a model-based approach has been used. A sophisticated system for liver surgery simulation has been developed at INRIA [6] which includes advanced $3 \mathrm{~d}$ interaction techniques. The Visible Human dataset was used for the creation of their model. Furthermore, experiences with a deformable volumetric model of the liver parenchyma have been described in [1]. With this model, cutting procedures can be simulated.

The use of the SURGERYPLANNER requires image processing with our HEPAVISION system which integrates the algorithms for liver and tumor segmentation, vessel segmentation, vessel analysis and liver segment approximation [4], [8-10]. For the liver and tumor segmentation a live wire segmentation and a modified watershed transform are available. For the analysis of the vessel systems the result of the vessel segmentation is skeletonized and transformed into a graph which represents the branching structure of the vessel system. The portal venous system and the hepatic veins are separated automatically. Furthermore, the individual branches of the vessel system are detected. Based on this analysis liver segments and subsegments are approximated. We compared different methods for this purpose. The easiest way to approximate liver segments is the nearest neighbor approach. With this approach each voxel of the liver parenchyma is supposed to be supplied by the portal venous branch which is nearest to it (in the Euclidean metric). The HEPAVISION system has been used in cooperation with 4 clinical partners.

\section{Interaction and Visualization Techniques}

In the image processing stage, carried out with HEPAVISION, the liver, the lesions and liver segments are identified and delineared. A tagged volume represents for each voxel to which object(s) it belongs and is used as input for the SURGERYPLANNER.

\subsection{Visualization of Anatomic and Pathologic Structures}

The SURGERYPLANNER uses the information which results from the image processing to allow the user to design a $3 \mathrm{~d}$ visualization. The user can select a subset of the identified objects and can assign different viewing parameters (e.g. colors) to each 
object (see Fig. 1 and Fig. 2). These objects are rendered in an OPENINVENTOR viewer with the usual facilities for camera control to explore the visualization. The SURGERYPLANNER is based on a volume renderer developed at MEVIS. The renderer provides various direct volume rendering (DVR) as well as surface rendering techniques. It turned out to be useful to present the segmented structures as surface rendering (including shading effects which reveal the spatial relations more clearly) and to embed the segmented structures in a DVR of the surrounding bones to provide the visual context to assess the viewing direction. The viewer used in the SURGERYPLANNER is a special viewer which extends the OPENINVENTOR facilities in two ways: it provides a shadow projection on a camera-fix plane for better perception of spatial relations and supports $3 \mathrm{~d}$ interaction with a SpaceBall. With these facilities users can recognize and explore spatial relations to become familiar with them.

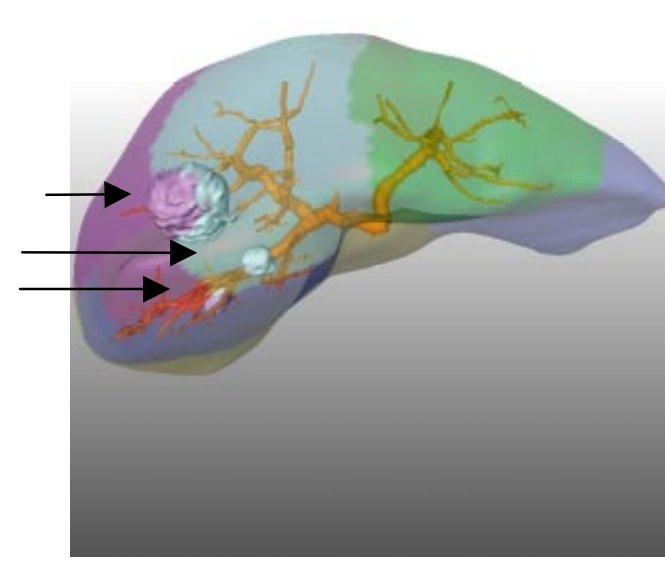

Fig. 1: Semi-transparent liver segments with three lesions (see arrows) and the portal venous system inside.

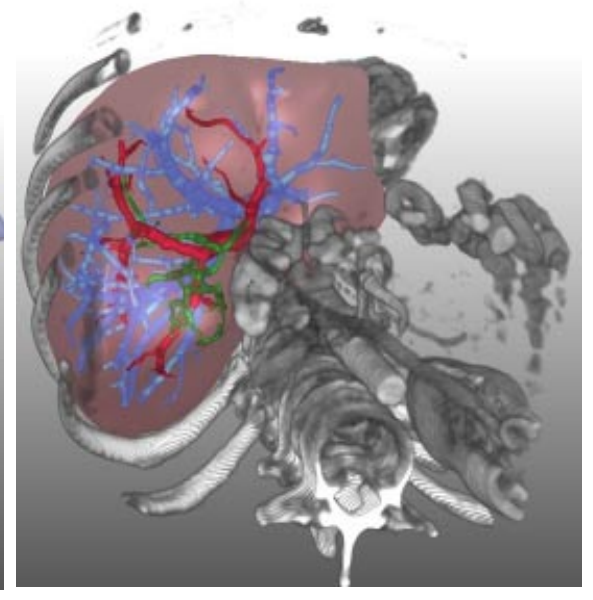

Fig. 2: The liver with arteries, veins and the portal vein embedded in the volumerendered surrounding.

\subsection{Cutting Arbitrary Regions}

As surgeons want to be able to try resection strategies we developed techniques to cut arbitrary regions from a volume. Efficient removal of arbitrary regions from a volume has been described in [11]. We follow a different approach using implicit functions to define resection regions analytically. A large variety of $3 \mathrm{~d}$ objects, like wedges and cylinders, can be conveniently defined with implicit functions.

The strategy of our algorithm is as follows: a convex resection region $R$ is defined by an implicit function. With a transformation $T$ the resection region is mapped into a discrete mask volume $M$, the size of which corresponds to the data volume $V$. For each voxel $v=\left\{v_{x}, y_{y}, v_{z}\right\}$ in $V$ the corresponding voxel $m=\left\{m_{x}, m_{y}, m_{z}\right\}$ in $M$ is TRUE if $m$ belongs to $T(R)$. In order to identify quickly the voxels which belong to $T(R)$, an additional data structure, a brick volume $B$, is introduced. In $B$ one item represents a brick of $M$ with the original size of $12 \times 12 \times 12$ voxel. $B$ is used to record which region 
in $M$ has been processed. Since $R$ is convex it is in most cases sufficient to check the vertices of a brick whether they belong to $T(R)$. If the test yields TRUE for all vertices the brick is filled iteratively. If the test yields FALSE for all vertices the whole brick is outside $T(R)$. Only if the test yields TRUE for some vertices and FALSE for others the brick is subdivided. The recursive subdivision may be finished by examining $3 \times 3 \times 3$ sized cubes (which are filled completely depending on the test of the central voxel) or by examing individual voxels. The latter high-quality mode is only applied when the user stops moving the resection tool. Like a recursive fill, the algorithm starts at a voxel inside of $T(R)$, considers its brick and recursively visits neighboring bricks (with subdivision if required) until no neighboring bricks are found which belong to $T(R)$.

With this approach the resection region is explicitly represented in $M$ and can be modified with morphologic image processing techniques (dilatation and erosion, close gaps). All voxels in $M$ belonging to $T(R)$ can be combined with boolean operations which is useful to include conditions which decide whether a voxel is actually drawn (this flexibility is used later for selective resections). In an 8-bit mask volume, 8 independent (and overlapping) resection regions can be managed which is sufficient for surgery planning. If $R$ is moved to $R^{\prime}$ two options are available: (1) the old mask $M$ is cleared and only $T\left(R^{\prime}\right)$ will be cut, or (2), $T(R)$ remains marked in $M$ as deleted. In the latter case the movement of $R$ defines a trace of arbitrary shape. Note, that this trace is not restricted to be convex, thus every possible resection can be defined.
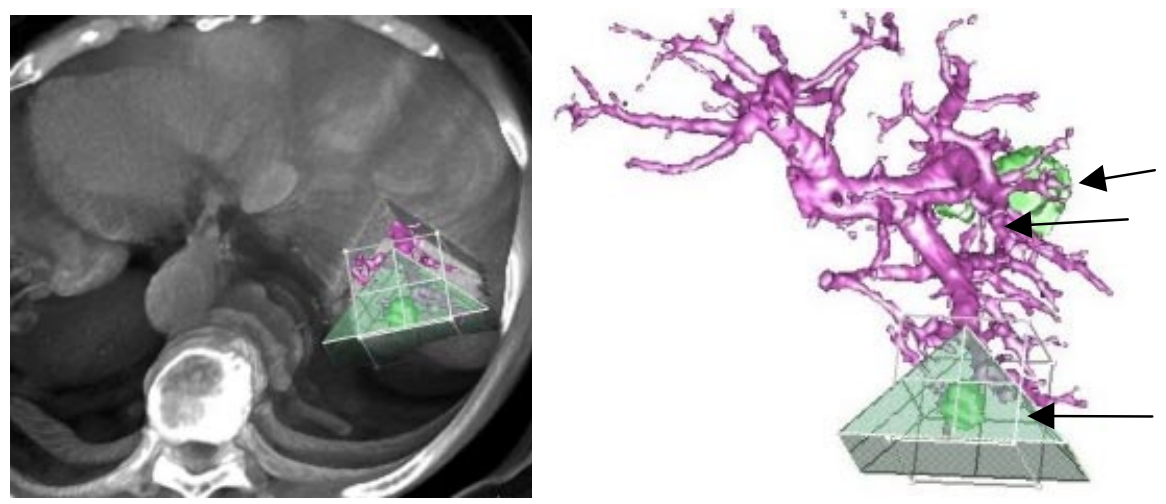

Fig. 3: A wedge-shaped resection tool is used for virtual resection in a CT data set. Both views are synchronized with respect to their viewing direction. The user can virtually resect in either view. In the left view the user gets an impression of the resection while the right view is used for orientation as it contains the vessels to be saved if possible and all lesions to be removed.

\subsection{Resection Tools for the Specification of Resection Regions}

For the trial of resections different resection tools are available: wegdes, clip planes, cylinders and spheres. Resection tools can be parameterized within appropriate dialoges and can be transformed in a $3 \mathrm{~d}$ visualization by means of manipulators provided by the Graphics Library OPENINVENTOR. The properties of a resection tool define its visualization (color, rendering style) and its initial orientation and position. Resection tools can be applied selectively to different structures so that certain objects are visible even if they belong to the resection region. Such visualizations have been 
succesfully used for anatomy education and are described in [12]. In the context of surgery planning it is helpful to see a lesion and major blood vessels in a region where other structures are removed (see Fig. 4). The user can thus assess the distance between the tumor and the boundary of the resection region and the vessels involved in this resection.

\subsection{Visualization of and Interaction with Resection Tools}

Concerning the usability of resection tools the visualization and the directmanipulative movement is crucial. Resection tools and their manipulators should be recognizable but should not occlude the resection region too heavily. A trade-off between these goals can be achieved with semi-transparent resection tools or tools which are visualized via their outlines or a combination thereof. The color must differ strikingly from that of the data values. Manipulators should be provided to support all transformation tasks: translation, rotation and scaling. After the size has been adjusted a resection tool is primarily translated and less often rotated. Based on this observation resection tools should be equipped with a manipulator dedicated for translation. The OPENINVENTOR Jack Manipulator is appropriate for this task. With this manipulator translations in the orthogonal planes are explictly supported. The Jack Manipulator can also be used to rotate an object. For this purpose a second mode exists with handles for translations temporarily hidden. The interactive resection is typically carried out in the following way: the user starts with a medium-sized resection tool and moves it through the data in order to remove a lesion. In this process, it is often necessary to rotate the visualization to evaluate what has been removed. After a rough boundary has been specified it is refined with a smaller-sized resection tool. Gaps in the resection region may be ignored as these may be filled automatically. A two-handed interaction with the system is possible with one hand to control the resection (with a $3 \mathrm{~d}$ input device) and the other to simultaneously control the camera (with a $2 \mathrm{~d}$ mouse).

\subsection{Synchronization of Different Views}

In the process of surgery planning a variety of different visualizations are generated: the viewing direction changes, different objects are visible, and resection tools are applied. For many visualization goals no single visualization is appropriate and even the interactive handling is insufficient, e.g. to compare different views under certain aspects. Therefore, it is crucial to have multiple views which can be flexibly parametrized. In the SURGERYPLANNER the user can freely add viewers which are named automatically. In an overview each viewer is represented by an icon and its name in order to switch quickly between different viewers (see Fig. 4).

In each viewer not only the viewing direction may be altered but also the subset of objects displayed, the appearance of objects and the resection tools applied. To support the comparison of different views, the user can define synchronizations between selected viewers. Viewers may be synchronized for example concerning the application of resections, or concerning rotations of the scene, or filter operations. Moreover, in every dialog which affects the content of a viewer it can be specified whether these settings shoul $\mathrm{d}$ be applied to all viewers (regardless whether synchronizations apply). 


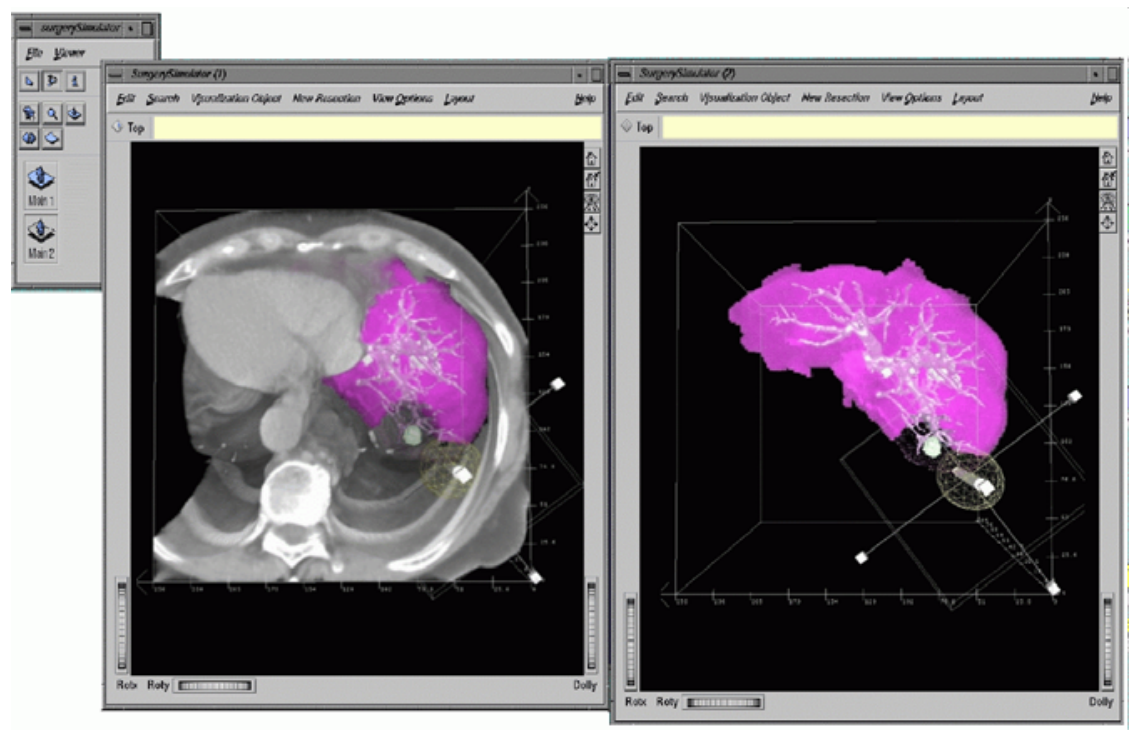

Fig. 4: Two synchronized views for the specification of a resection. The left view provides the visual context (bones, heart), while the right view (with the unoccluded liver) is used to move the resection tool.

\section{Suggestions for Optimal Resections}

With direct-manipulative resection tools the user has full control to flexibly develop and explore resection strategies. However, some experience with $3 \mathrm{~d}$ interaction and a few minutes to specify a resection are required. An alternative approach to preoperative planning is to let the system emphasize what should be removed given a lesion and the location and branching structure of the portal venous system and taking into account how the resection influences vessels and the supplied liver parenchyma. We developed such a high-level support based on the scenario described in Sect. 2: starting from a selected lesion and a given tumor free margin, the involved parts of the vessel system are identified and the supplied liver parenchyma is calculated.

We started to investigate this approach by working with CT images of 8 corrosion casts of human cadavers which have been kindly supplied by PD Dr. Fasel (University Genf, Department for Morphology). With these models the portal vein could be reconstructed up to the $6^{\text {th }}$ branching order - the overall length of the extracted vessels is an order of magnitude larger than in clinical data. In these models we included a sphere with $1 \mathrm{~cm}$ diameter as model of a focal lesion and calculated the liver parenchyma to resect for different safety margins (see Fig. 5 and Fig. 6). Safety margins are defined by a sequence of dilatations of the lesion. The calculation of resction regions also considers large hepatic veins which should not be damaged. For each corrosion cast this calculation has been carried out for 1500 tumor locations summarized in a video which we carefully discussed with our clinical partner Prof. Oldhafer. It turned out that the resection regions proposed by the system correspond well to surgical practice in shape and size. In the large majority of the cases, the high- 
lighted regions could have been resected. In a second experiment we analyzed a clinical data set with 3 lesions. This CT data set allowed us to extract $4^{\text {th }}$ order brancing of the portal vene. With this prerequisite suggestions for resections could be defined (see Fig. 7 and Fig. 8).

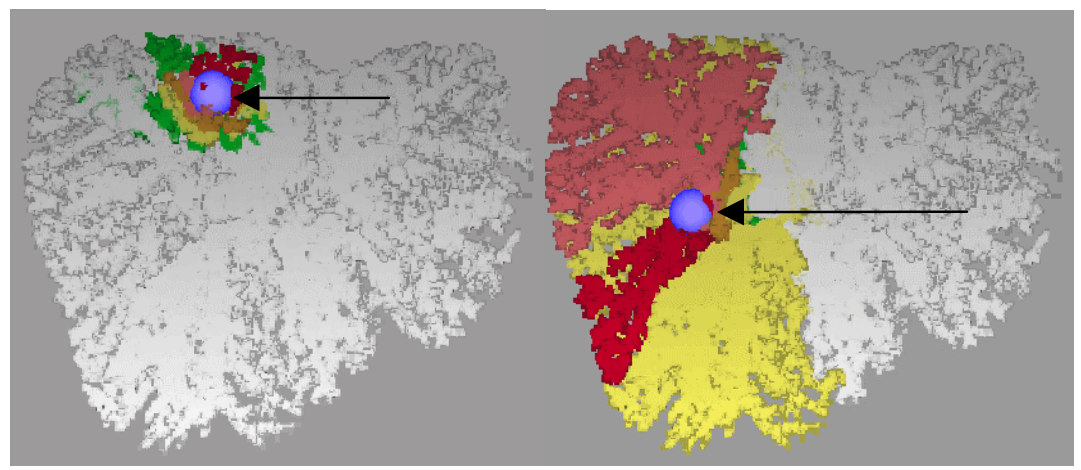

Fig. 5: A sphere has as a model for a focal lesion been moved through a model of a corrosion cast with the portal vein. The vessels not involved in the resection are displayed white. Different colors indicate the vessels involved in the resection with different margins $(0.5 \mathrm{~cm}, 1.0$ $\mathrm{cm}, 1.5 \mathrm{~cm}, 2.0 \mathrm{~cm}, 2.5 \mathrm{~cm})$.

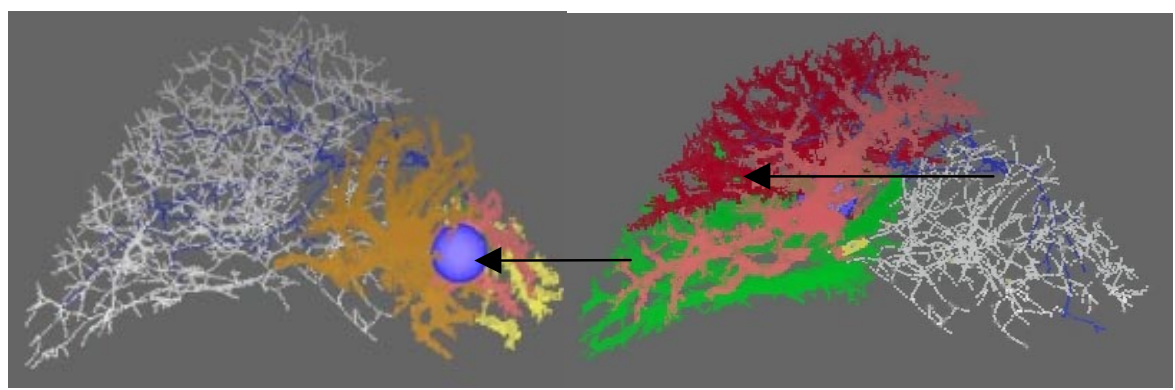

Fig. 6: The same experiment as in Fig. 5 with a model with hepatic veins and the portal venous system. The vessels not involved in the suggested resection are displayed skeletonized to better reveal the branching pattern. Different colors again indicate the vessels involved in the resection with different margins. The hepatic vein is considered in the suggested resection.

The way by which the tumor appears in a CT image and thus the size and shape of the segmented tumor is not perfectly reliable. Moreover, the surgeon cannot exactly follow a planned resection. Suggestions for resections have to consider the limited accuracy of the data and the procedure. Therefore the calculation is carried out for different margins: the red one being the inner region which must be definitely resected, the other colors representing more distant zones. The visualization thus indicates the risk involved in the procedure for the damage of major blood vessels (which are in the orange or yellow region). The resection regions suggested can be fine-tuned interactively. For this purpose the user selects a region which is subsequently transformed in the mask volume for manipulation with a resection tool. 


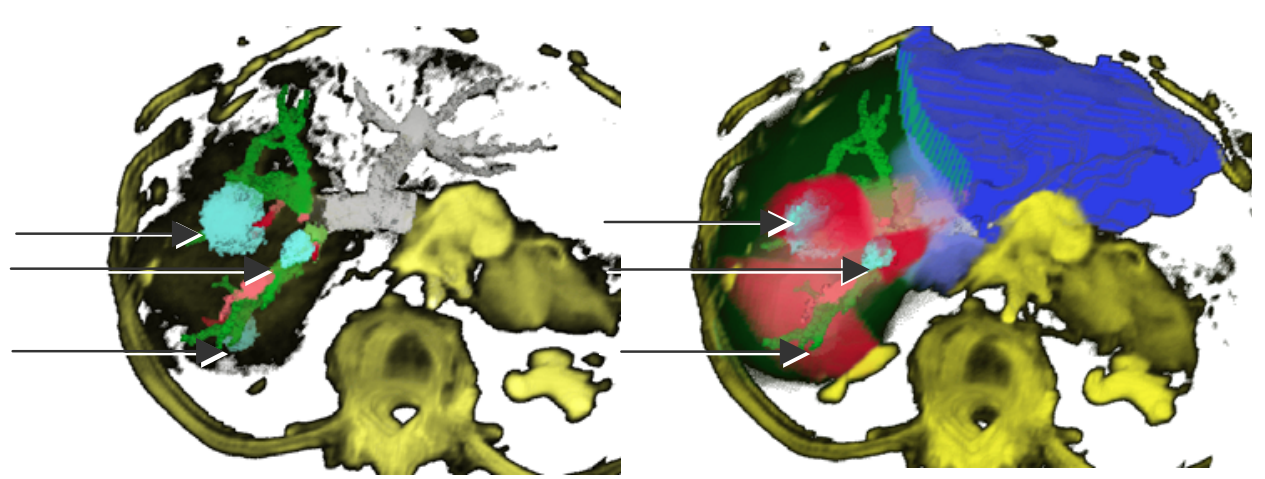

Fig. 7: In a case with three lesions (see the Fig. 8: The regions supplied by the vessels arrows) the dark vessels are involved in the removal whereas gray vessels are not affected.

involved in the resection are rendered semitransparent. The resection of the left hemi-liver ( $49,4 \%$ of the liver parenchyma) is indicated.

\section{Conclusion and Future Work}

The SURGERYPLANNER provides a reliable base to aid in the complex decision making process concerning the operability of patients with focal liver lesions. For this purpose a flexible $3 \mathrm{~d}$ visualization and resection tools for the preoperative planing and analyis of a resection are provided. An efficient algorithm for cutting arbitrary regions is used for interactive resection. With these methods, several patients have been virtually resected using the data processed with our HEPAVISION system. The handling of the resetion tools turned out to be difficult, without stereo rendering. Once a resection is defined, however, it helps considerably to judge an operation. The selective application of resection tools and synchronization mechanisms are essential for the usefulness of the system. Based on established guidelines and a user-defined margin suggestions for resections are generated as an alternative approach to surgery planning. With this unique feature the risk for long-term survival can be estimated. Interactive resections and suggestions by the system can be combined flexibly: either the user refines interactively a resection area suggested by the system or the system analyzes a user-defined resection area. The combination of flexible interaction techniques with high-level support is not bound to liver surgery but can be applied, e.g. to lung surgery.

The support provided by the SURGERYPLANNER is considerable but also has limitations. The system does not guide the surgeon during the operation. Intraoperative support, as in neurosurgery, is difficult to realize in liver surgery due to the surmounting problems with intraoperative registration. Moreover, the decision whether a patient can tolerate a resection is not fully supported. In particular, primary liver carcinoma are often inoperable due to the extent of liver cirrhosis which accompanies the cancer disease. In some cases patients are inoperable due to lesions outside the liver or due to the genral state of the patient. The SURGERYPLANNER can only be used to decide whether patients are locally operable (that is whether liver lesions might be resected).

Future work will concentrate on the following problems: an in-depth evaluation in the preoperative use of the SURGERYPLANNER, the use of MR data which requires 
to fuse several MR sequences which selectively highlight the relevant structures and the refinement of the suggestions for resections. In rare cases where a lesion is centrally located at the back side $(\approx 5$ percent $)$ the suggestions generated require to resect the liver ex-situ (which is an extremely demanding procedure carried out only at a few hospitals). In these cases our suggestions should consider the access to the resection region to find out whether an alternative access is possible. Currently, the user can treat these cases with interactive resection.

\section{Acknowledgments}

We would like to thank our colleagues A. Schenk, D. Böhm and Dr. T. Schindewolf for their contribution to the HEPAVISION system. Furthermore, we thank Prof. Galanski, Dr. Högemann, and Dr. Stamm (Department of Diagnostic Radiology, Medical School Hannover) as well as Prof. Klose and his team (Department of Diagnostic Radiology, Philipps-University Marburg) for fruitful discussions. Fig. 1, 3, 5, 6, 8 and 9 are based on data kindly provided by Prof. Klose. Fig. 2 and Fig. 4 are renditions of datasets kindly provided by Prof. Galanski. We thank Felix Ritter, University of Magdeburg, who provided us with a special viewer which supports shadow projection and the use of $3 \mathrm{~d}$ input devices. We acknowledge the German Reseach Council (DFG) for support of our project under the number PE 199/9-1.

\section{References}

1. Boux de Casson F, and Laugier C (1999) "Modeling the Dynamics of a Human Liver for a Minimally Invasive Surgery Simulator", Proc. of MICCAI, Springer, LNCS, Vol 1679, pp. 1156-1165

2. Couinaud, C. Le foie - Etudes anatomiques et churgicales, Paris, Masson, 1957

3. Dokladal, P, Lohou C, Perroton L, and Bertrand G (1999) "Liver Blood Vessel Extraction by a 3d toplogical approach", Proc. of MICCAI (Cambridge, UK, 19.-22. Sept.), Springer, LNCS, Vol 1679, pp. 98-104

4. Fasel JHD, Selle D, Gailloud P et al. (1998) "Segmental Anatomy of the Liver: Poor Correlation with CT", Radiology, Vol 206 (1), pp. 151-156

5. Fishman, EK, Kuszyk BS, Heath DG, Gao L, and Cabral B (1996) "Surgical Planning for Liver resection", IEEE Computer, January 1996, pp. 64-72

6. Marescaux J, Clement JM, Tassetti V et al. (1998) "Virtual reality applied to Hepatic Surgery Simulation: The Next Revolution", Annals of Surgery, Vol 228 (5), pp. 627-634

7. Mazziotti A and Cavallari A (1997) Techniques in Liver Surgery, Greenwich Medical Media

8. Oldhafer KJ, Högemann D, Stamm G, Raab R, Peitgen HO, and Galanski M (1999) "Dreidimensionale Visualisierung der Leber zur Planung erweiterter Leberresektionen", Der Chirurg, Vol 70, Springer, pp. 233-238

9. Schenk, A, Prause G and Peitgen HO (2000) "Efficient Semiautomatic Segmentation of 3D Objects in Medical Images", Proc. of MICCAI (Pittsburgh, 11.-14. Okt.), Springer, LNCS (to appear in this volume)

10. Selle D, Schindewolf T, Evertsz CJG, and Peitgen HO (1999) "Quantitative analysis of CT liver images", Excerpta Medical International Congress, Vol 1182, Elsevier, pp. 435-444

11. Udupa JK and Odhner D (1991) "Fast Visualization, Manipulation and Analysis of Binary Volumetric Objects", IEEE Comput. Graphics Appl., Vol 11 (6), pp. 53-62

12. Tiede, U, Bomans, M, Höhne KH et al. (1993) "A computerized three-dimensional atlas of the human skull and brain”, Am. J. Neuroradiology, Vol 14 (3), pp. 551-559 